\title{
Knee Joint Load Reduction by using Lateral Wedge Insole in Different Body Mass Index Level Females
}

\author{
Chitchanok Nutalaya $^{1^{*}}$, Montakarn Chaikumarn $^{2^{*}}$ \\ ${ }^{1}$ M.Sc. (Sports Sciences in Biomechanics) \\ Department of Physical Therapy Faculty of Allied Health Sciences \\ Chulalongkorn University, Bangkok, Thailand \\ ${ }^{2}$ Assistant Professor, Ph.D. (Ergonomics) \\ Department of Physical Therapy Faculty of Allied Health Sciences \\ Chulalongkorn University, Bangkok, Thailand \\ *Corresponding author's email: montakarn.c [AT] chula.ac.th
}

\begin{abstract}
Background: The higher body mass index is a risk factor that associated with onset and progression of knee osteoarthritis by increasing the knee adduction moment. Lateral wedge insoles are used for reducing the knee adduction moment which may prevent the occurrence of knee osteoarthritis in healthy population.
\end{abstract}

Purpose: The purpose of this study was to assess the effectiveness of the lateral wedge insole among normal, overweight, obese I and obese II female while walking.

Methods: A cross-sectional experimental design study was used to investigate 80 females who aged between $18-40$ years using 3-D Motion Analysis. The peak external knee adduction moment was collected in walking shoes and walking shoes with 5-degree lateral wedge insoles conditions. The significant difference was determined using Paired-T test (p $=0.05$ ).

Results: The peak external knee adduction moment was significantly decreased by the effect of shoes with lateral wedge insoles in normal, overweight and obese I (22.12\%, $18.34 \%$ and 24.15\%). However, it did not change in the obese II.

Conclusion: The peak external knee adduction moment was decreased by the immediate effect of lateral wedge insoles. It may prevent the onset of knee osteoarthritis in normal, overweight, and obese I. Further study should investigate long term effect of lateral wedge insoles on the knee joint load and the effect of dose in higher body mass index.

Keywords--- Lateral wedge insole, Knee joint load, Walking, Obesity, Female

\section{INTRODUCTION}

According to a World Health Organization (WHO) report, more than 1.6 billon adults are overweight [1]. Obesity is a metabolic disease of global concern and a strong risk factor for several diseases including knee osteoarthritis [2] The prevalence of obesity in Thailand has doubled over the past two decades. Data from three consecutive National Health Examination Surveys (NHES) have shown a secular trend, as the prevalence of obesity with a body mass index $\geq 25 \mathrm{~kg} / \mathrm{m}^{2}$ among adults increased from $13.0 \%$ in men and $23.2 \%$ in women in 1991 to $22.4 \%$ and $34.3 \%$ in 2004, respectively [3]. Increased body mass index contributes to a substantially increased risk of knee osteoarthritis. The magnitude of obesity as a risk factor of osteoarthritis is significantly stronger in women than men $[4,5]$. From previous study, it revealed that the peak external knee adduction moment associated with the risk of knee osteoarthritis was significantly increased when the body mass index was higher in healthy female [6].

There is growing interest in the role of the external knee adduction moment in the pathogenesis of knee pain and osteoarthritis $[7,8]$. The external knee adduction moment is produced by the ground reaction force passing medial to the center of the knee joint during gait and is primarily derived from the product of the resultant ground reaction force in the frontal plane and its moment arm (the perpendicular distance from the ground reaction force to the knee joint center of rotation). The external knee adduction moment tends to adduct the knee into a varus position and is significantly correlated with disease severity [9]. Moreover, the thickness of the medial tibial cartilage is related with the peak knee adduction moment and knee adduction moment impulse that represents the dynamic knee joint load during walking [10].

The orthosis has been investigated as potential non-invasive management of knee osteoarthritis for reducing the biomechanical factor that associate with the progression of the symptom. [11-16]. More specifically, lateral wedging has 
been used with the goal of reducing the symptoms associated with medial knee osteoarthritis, hypothetically by reducing the peak external knee adduction moment $[16,17]$. If using the lateral wedge insoles are associated with decreased the knee joint load during walking and other physical activities, these may be useful in the management of the symptoms of patients with medial knee osteoarthritis and potentially to reduce the risk of future osteoarthritis in individuals at increased risk, such as those with knee injuries or who are obese. However, the effectiveness of lateral wedge insoles on knee joint load parameters such as the external knee adduction moment and knee adduction moment impulse in various body mass index is unclear.

Therefore, we focused our investigation on the effect of lateral wedge insoles on the kinetic parameters of the external knee adduction moment and knee adduction moment impulse of each body mass index level during the stance phase of walking. The primary hypothesis was that shoes with 5-degree lateral wedge insoles would reduce the knee joint load.

\section{METHODS}

Eighty females who aged between 18 to 40 years, were right leg dominant participated by convenience sampling in this study. The sample size was calculated using software $G^{*}$ Power version 3.0.10. The International Obesity Task Force (IOTF) and the Steering Committee of the Regional office for the Western Pacific Region of WHO (WPRO) recommend that the overweight specification for an adult in Asia is when the body mass index exceeds $23.0 \mathrm{~kg} / \mathrm{m}^{2}$ and that for obesity when the body mass index exceeds $25.0 \mathrm{~kg} / \mathrm{m}^{2}$. This Asian classification was adopted in this study. The inclusion criteria were included a body mass index of 18.5 to $22.9 \mathrm{~kg} . / \mathrm{m}^{2}$ (normal), 23 to $24.9 \mathrm{~kg} / \mathrm{m}^{2}$ (overweight), 25 to $29.9 \mathrm{~kg} / \mathrm{m}^{2}$ (obese I) and over $30 \mathrm{~kg} / \mathrm{m}^{2}$ (obese II), sedentary life style, free of lower extremity injuries affecting gait and with no knee pain, no previously diagnosed with or experienced knee osteoarthritis, or medical treatment for knee osteoarthritis related symptoms, weight change $<2.5 \mathrm{~kg}$ net during the previous 3 months, normal foot morphology, static knee alignment axis in frontal plane (valgus and varus) less than 10 degrees. All participants were informed about the objective, process of testing, the equipment components and safety features. Participants were informed about the procedures and signed informed consent in accordance with the Ethics Review Committee for Research Involving Human Research Subjects, Health Science Group, Chulalongkorn University.

\subsection{Experimental protocol}

All of the practice and testing trials were performed at the Biomechanical Laboratory, Department of Physical Therapy, Faculty of Allied Health Sciences, Chulalongkorn University. The biomechanics data were collected using an eight camera Motion Analysis System (Motion Analysis Corp., Santa Rosa, CA) with a flash rate of 120 Hz. The cameras were synchronized with the BERTEC strain gauge technology force platform, which were set to have a sampling frequency of 1,200 Hz. Cortex version 2.5 software was used for calibration of capture volume, tracking and identifying marker locations in a calibrated 3D space and post processing 4 tools for tracking, editing, and preparing the data for other packages. Then the data were processed with a clinical gait evaluation module by OrthoTrak 6.6 Gait Analysis Software. Reference and volume calibrations were performed before experimental trial. To track the motions, the 29 spherical retroreflective markers were placed on anatomical landmarks and segments following the Helen Hayes marker set by the researcher who had over 10 years' experience identifying anatomical landmarks [18]. The reflective markers were attached with adhesive tape at the center top, front and back of head, acromion process, lateral epicondyle of humerus, center between medial and lateral styloid processes, the right medial border of scapular to be offset, the L5-Sacral interface, anterior superior iliac spine, the midpoint of thigh, medial and lateral femoral condyle, midpoint of shank, medial and lateral malleolus, calcaneus and the center between $2^{\text {nd }}$ and $3^{\text {rd }}$ metatarsal were located of the markers. These markers were defined as anatomical markers to define the anatomical reference frames and center of rotation of the joints.

Participants were asked to perform a static quiet standing position with 90-degree abduction shoulders for detect the 29 reflective markers. Then, the four medial markers of the knees and ankles were removed to prepare for the walking trial. Participants were allowed to walk with shoes (Reebok ${ }^{\mathrm{TM}}$ Model V53843), and shoes with lateral wedge insoles (5 and 70 Shore A hardness, Salfordinsole ${ }^{\mathrm{TM}}$, UK) [19]. The order of the shoe conditions was simple randomized. Participants were required to walk along a $10 \mathrm{~m}$. walkway and given enough time to warm up. A five-minute rest between five successful trials was performed to prevent muscle fatigue. Five successful trials were defined those in which the subjects walk with complete contact on the force plate of each shoe conditions were collected [20].

The data were filtered by a Butterworth $4^{\text {th }}$ order bi-directional filter for the kinematic data using $6 \mathrm{~Hz}$ and $25 \mathrm{~Hz}$ for force data. Cortex version 2.5 and OrthoTrak Gait Analysis version 6.6 software were used in post process to define the relevant data including kinetics: peak vertical ground reaction forces (PVGRF: BW), peak external knee adduction moment (PEKAM: $\mathrm{Nm} / \mathrm{kg}$ and $\mathrm{Nm}$ ) and knee adduction angular impulse (KAAI: $\mathrm{Nm} / \mathrm{kg}_{*} \mathrm{~s}$ ) during the early stance phase. The inverse dynamic solution was used to calculate the external knee adduction moment. 


\subsection{Statistical analysis}

Statistical analysis was performed using SPSS 17.5 for Windows. The level of significance was set at p-value $\leq$ 0.05. Subject characteristic data were expressed as mean and standard deviation (SD) values. The peak vertical ground reaction forces (PVGRF: BW), peak external knee adduction moment (PEKAM: Nm/kg and Nm) and knee adduction angular impulse (KAAI: $\mathrm{Nm} / \mathrm{kg}_{*} \mathrm{~S}$ ) during the early stance phase data were expressed as mean and standard error of mean (SEM) values. The normal distribution of data was tested using the Kolmogorov-Smirnov test. The \% changes between the walking conditions of each group were calculated follow the equation and the mean significant difference were tested by the Paired T-test.

$$
\% \text { Changes }=\left(\frac{\bar{X}_{\text {shoe }}-\bar{X}_{\text {shoe with lateral wedge insole }}}{\bar{X}_{\text {shoe }}}\right) \times 100
$$

\section{RESULTS}

The 80 participants' demographic and anthropometric characteristics were presented in Table 1. There was no significant difference between groups as regarding in age, height, and leg length. There were significant differences between the groups in that of the weight, body mass index, waist circumference, hip circumference, waist-hip ratio, and percent body fat which were higher in the overweight, obese I and obese II groups than the normal group. Moreover, the knee circumference and Q angle showed an increase among the normal, overweight, obese I and obese II groups.

Table 1: Demographic and anthropometric characteristics of eighty female participants.

\begin{tabular}{|c|c|c|c|c|c|c|c|c|c|}
\hline \multirow{2}{*}{ Parameter } & \multicolumn{2}{|c|}{ Normal $(n=20)$} & \multicolumn{2}{|c|}{ Overweight $(\mathrm{n}=20)$} & \multicolumn{2}{|c|}{ Obese I $(\mathrm{n}=20)$} & \multicolumn{2}{|c|}{ Obese II $(n=20)$} & \multirow{2}{*}{$P$ value } \\
\hline & Mean & SD & Mean & SD & Mean & SD & Mean & SD & \\
\hline Age (Years) & 24.5 & 3.43 & 26.6 & 5.71 & 29.25 & 6.13 & 26.85 & 6.23 & 0.065 \\
\hline Weight (kg.) & 51.1 & 2.89 & 59.94 & 4.1 & 69.33 & 6.37 & 86.99 & 9.88 & $0.000^{*}$ \\
\hline Height (cm.) & 159.59 & 4.29 & 157.93 & 5.07 & 157.43 & 5.75 & 160.7 & 6.13 & 0.199 \\
\hline BMI $\left(\mathrm{kg} \cdot \mathrm{m}^{-2}\right)$ & 20.12 & 1.21 & 23.96 & 0.66 & 27.87 & 1.35 & 33.68 & 3.81 & $0.000^{*}$ \\
\hline Right leg length $(\mathrm{cm})$. & 81.4 & 3.62 & 79.3 & 4.24 & 78.58 & 4.09 & 80.91 & 2.88 & 0.064 \\
\hline Waist circumference $(\mathrm{cm})$. & 69.93 & 4.67 & 78.08 & 4.33 & 85.58 & 4.74 & 97.04 & 8.76 & $0.000 *$ \\
\hline Hip circumference $(\mathrm{cm})$. & 90.05 & 3.8 & 98.2 & 3.88 & 103.18 & 4.83 & 114.73 & 6.79 & $0.000 *$ \\
\hline Waist Hip ratio & 0.78 & 0.04 & 0.8 & 0.06 & 0.83 & 0.05 & 0.85 & 0.07 & $0.000^{*}$ \\
\hline Right knee joint circumference $(\mathrm{cm})$. & 33.69 & 1.39 & 36.93 & 1.7 & 37.75 & 2.39 & 41.93 & 2.88 & $0.000 *$ \\
\hline Right Q angle (degrees) & 10.35 & 1.09 & 11.3 & 1.78 & 11.55 & 1.96 & 12.45 & 1.9 & $0.003 *$ \\
\hline Percent body fat (\%) & 28.05 & 3.86 & 30.95 & 3.29 & 34.83 & 3.91 & 37.81 & 4.83 & $0.000 *$ \\
\hline
\end{tabular}

NOTE: * significantly different between groups $(P$-value $<0.05)$ 
Table 2: Kinetic parameters during walking with corresponding statistical findings

\begin{tabular}{|c|c|c|c|c|c|c|c|}
\hline \multirow{2}{*}{ Group } & \multirow{2}{*}{ Parameter } & \multicolumn{2}{|c|}{ Shoe only } & \multicolumn{2}{|c|}{ Shoe with insole } & \multirow{2}{*}{$\begin{array}{l}\% \\
\text { Changes }\end{array}$} & \multirow{2}{*}{$P$-value } \\
\hline & & Mean & SEM & Mean & SEM & & \\
\hline \multirow{4}{*}{$\begin{array}{l}\text { Normal } \\
(\mathrm{n}=20)\end{array}$} & Peak VGRF (BW) & 1.16 & 0.04 & 1.15 & 0.04 & 0.86 & 0.23 \\
\hline & Peak external knee adduction moment $(\mathrm{Nm} / \mathrm{kg})$ & 0.3 & 0.03 & 0.30 & 0.03 & 0.00 & 1.00 \\
\hline & Knee adduction angular impulse $\left(\mathrm{Nm} / \mathrm{kg}_{*} \mathrm{~s}\right)$ & 0.11 & 0.02 & 0.11 & 0.01 & 0.00 & 1.00 \\
\hline & Peak external knee adduction moment (Nm) & 13.79 & 1.29 & 10.74 & 1.31 & 22.12 & $0.027 *$ \\
\hline \multirow{4}{*}{$\begin{array}{l}\text { Overweight } \\
(\mathrm{n}=20)\end{array}$} & Peak VGRF (BW) & 1.12 & 0.03 & 1.10 & 0.03 & 1.79 & 1.00 \\
\hline & Peak external knee adduction moment (Nm/kg) & 0.25 & 0.03 & 0.24 & 0.02 & 4.00 & 0.10 \\
\hline & Knee adduction angular impulse $\left(\mathrm{Nm} / \mathrm{kg}_{* \mathrm{~s}}\right)$ & 0.09 & 0.01 & 0.09 & 0.01 & 0.00 & 0.47 \\
\hline & Peak external knee adduction moment (Nm) & 15.92 & 1.44 & 13.00 & 1.46 & 18.34 & $0.034 *$ \\
\hline \multirow{4}{*}{$\begin{array}{l}\text { Obese I } \\
(n=20)\end{array}$} & Peak VGRF (BW) & 1.09 & 0.03 & 1.09 & 0.02 & 0.00 & 1.00 \\
\hline & Peak external knee adduction moment $(\mathrm{Nm} / \mathrm{kg})$ & 0.21 & 0.03 & 0.20 & 0.03 & 4.76 & 1.00 \\
\hline & Knee adduction angular impulse $\left(\mathrm{Nm} / \mathrm{kg}_{*} \mathrm{~s}\right)$ & 0.07 & 0.01 & 0.07 & 0.01 & 0.00 & 0.46 \\
\hline & Peak external knee adduction moment $(\mathrm{Nm})$ & 18.26 & 1.69 & 13.85 & 1.9 & 24.15 & $0.024 *$ \\
\hline \multirow{4}{*}{$\begin{array}{l}\text { Obese II } \\
(n=20)\end{array}$} & Peak VGRF (BW) & 1.11 & 0.01 & 1.11 & 0.01 & 0.00 & 1.00 \\
\hline & Peak external knee adduction moment $(\mathrm{Nm} / \mathrm{kg})$ & 0.25 & 0.02 & 0.24 & 0.02 & 4.00 & 0.09 \\
\hline & Knee adduction angular impulse $\left(\mathrm{Nm} / \mathrm{kg}_{*} \mathrm{~s}\right)$ & 0.07 & 0.01 & 0.07 & 0.01 & 0.00 & 1.00 \\
\hline & Peak external knee adduction moment (Nm) & 21.88 & 2.36 & 21.83 & 1.97 & 0.23 & 1.00 \\
\hline
\end{tabular}

NOTE: * significantly different between walking conditions $(P$-value $<0.05)$

Table 2. presented the knee kinetics of the four body mass index groups during walking. The 5-degree lateral wedge insole had significantly reduced the peak external knee adduction moment $(\mathrm{Nm})$ in normal $(22.12 \%)$, overweight (18.34\%), and obese I (24.15\%) groups. However, there was no significant difference for the peak external knee adduction moment between shoe and shoes with insoles walking in only the obese II group $(0.23 \%)$ as presented in Figure 1. Moreover, there was no significant difference in peak vertical ground reaction force, knee adduction moment impulse and normalized peak external knee adduction moment between the walking conditions in all groups 
(A)

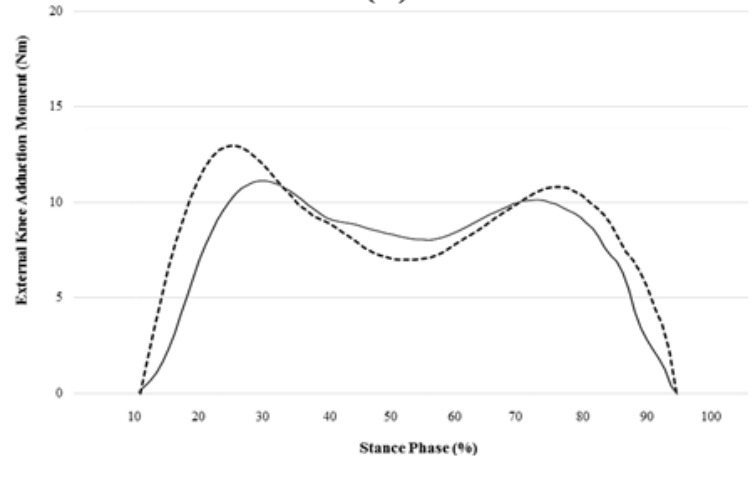

(C)

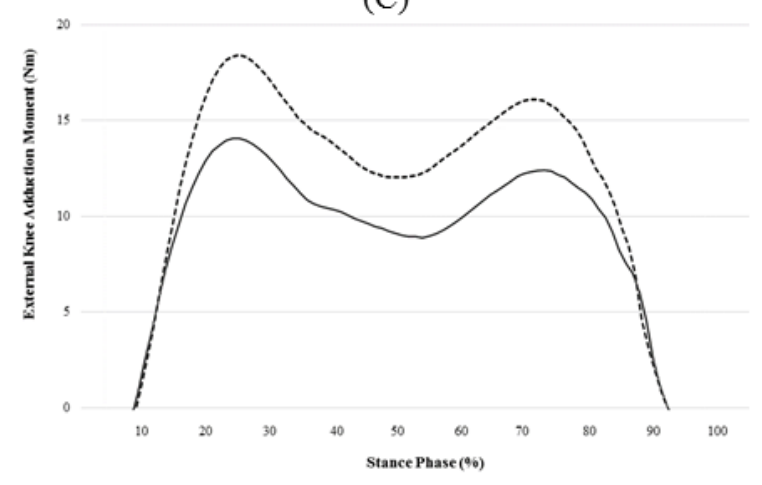

(B)

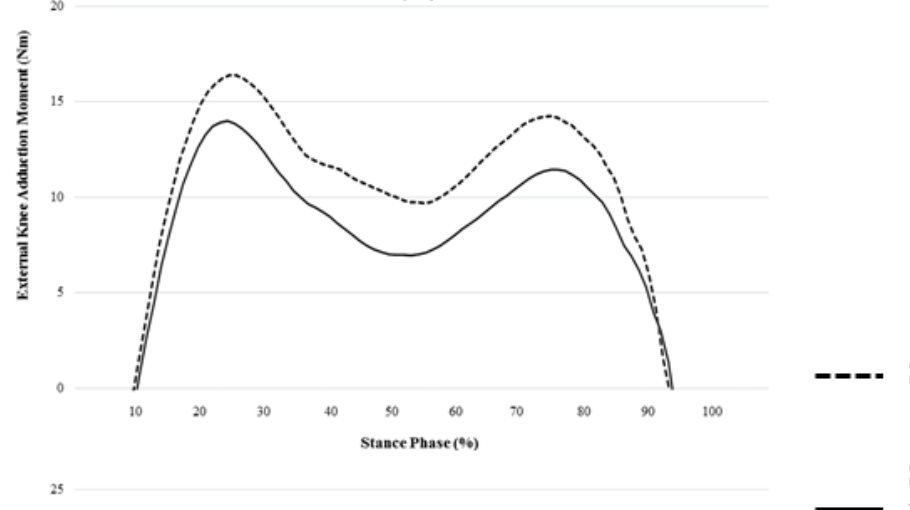

(D)

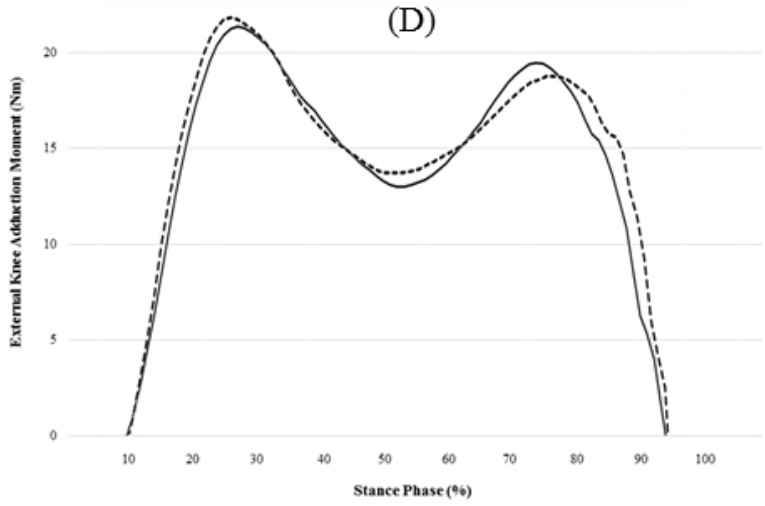

Shoe

Shoe with lateral wedge insole

Figure 1: The average external knee adduction moment $(\mathrm{Nm})$ in the 2 walking conditions during stance phase of each groups.

$(\mathrm{A}=$ normal, $\mathrm{B}=$ overweight, $\mathrm{C}=$ obese $\mathrm{I}$, and $\mathrm{D}=$ obese $\mathrm{II})$

\section{DISCUSSION}

The purpose of this study was to determine the effect the 5-degree lateral wedge insole could decrease the kinetic risk factors for knee osteoarthritis in normal, overweight, obese I and obese II women. Our study supports the primary hypothesis that the 5-degree lateral wedge insoles can reduce the knee joint load. Only the peak external knee adduction moment in lateral wedge insoles condition was significantly lower than those in shoe condition during walking in normal $(22.12 \%)$, overweight $(18.34 \%)$, and obese I (24.15\%).

As the recent studies, the evidence showed that lateral wedge insoles could reduce the peak external knee adduction moment in normal [19, 21] and obese participants with and without knee osteoarthritis [17, 22-26]. Higher body mass index are associated with joint tissue breakdown in knee osteoarthritis, attributable to greater total accumulation of load and altered joint loading, as well as reduced tissue tolerance due to inflammation [27]. The peak knee adduction moment play role in biomechanical risk factors of knee osteoarthritis is increase in higher body mass index [6,27] .This factor is important modifiable prevent and treatment targets in knee osteoarthritis, as each has the potential to affect joint tissue integrity by modulating compressive forces across the knee [6, 27].

Lateral wedged insoles are one of the gait modifications, which potentially delay the progression of medial knee osteoarthritis by inducing the lateral shift of the center of pressure in the foot. This shifts the frontal plane ground reaction force vector towards the knee joint center. Together with the more vertically oriented ground reaction force, this serves to reduce the moment arm of the knee adduction moment cause of the central mechanism explaining the load-reducing effect of lateral wedge insoles $[23,26]$. From our study, the 5-degree lateral wedge insole that immediately effected on reducing of the peak external knee adduction moment result to reduce the knee joint load may use for preventing risk of the knee osteoarthritis in women who were normal, overweight, and obese I. The long-term effect of using the lateral wedge insole on knee joint load reduction need to be studied for finding the benefit of application to reduce knee joint load and prevent the future knee osteoarthritis. 
The result from this study demonstrated that the peak external knee adduction moment did not change in the obese II group during walking. As can be seen from the mechanism of the action of the lateral wedge insoles on knee joint load, the degree of the lateral wedge insoles could have effect on reducing the knee to ground reaction force lever arm by increasing the lateral shift of the centre of pressure [23]. The 5-degree lateral wedge insole could reduce the knee joint load parameter among normal subjects [19]. However, in the obese II group, the 5-degree lateral wedge insole might be not appropriate for decreasing knee joint load by reduction of lever arm. A recent study recommended a 4-degree lateral wedge insole could reduce the knee joint load and the higher lateral wedging degree could further reduce the peak external knee adduction moment but be more uncomfortable (over 8 degrees) among the normal group [21]. Our findings suggested that the appropriate dose of lateral wedge insole for reducing the knee joint load in the higher body mass index should be interested to further studied, however the degree of lateral wedge insoles should be of concern for comfortable in application.

Investigations of joint loading in the knee typically normalize the knee adduction moment to global measures of body size such as body weight and height to allow comparison between individuals. However, such measurements may not reflect the knee architecture that affects the force acting upon the articular surface. Recent studies found that normalized peak external knee adduction moment was not sensitive to osteoarthritis severity; however, the non-normalization technique was superior at distinguishing between osteoarthritis severities [28-30]. In the present study, we presented the results both of normalized and non-normalized peak external knee adduction moment.

The lateral wedge insole may reduce the occurrence of knee osteoarthritis in high risk person such as female with high BMI level, which may decrease the joint load that is knee osteoarthritis risk factor. Therefore, the lateral wedge insoles may be an effective orthosis to manage the risk factors attributing to the onset and persistence of knee osteoarthritis in females.

A major strength of this study is that the knee joint load while walking was objectively and continuously assessed using a 3-D motion analysis system. However, this study has several methodological limitations that are noteworthy. First, the use of convenience sample restricts the external validity of this study. Thus, generalization of the results from this study to other working populations should be interpreted with caution. Second, the present study only investigated the knee kinetics in healthy females. Change in knee kinetics during walking in those with knee osteoarthritis may not correspond to that of an asymptomatic population. Thus, extrapolation of these results to people with knee osteoarthritis should be made with caution. Further research is required to examine the effect of lateral wedge insoles on knee kinetics in patients with knee osteoarthritis. Last, owing to the cross-sectional design of the present study, it is not possible to establish a causal relationship between exposure and outcome. Only the association between exposure and outcome was examined. Therefore, future study with a prospective study design is required to validate the findings of this study.

\section{CONCLUSIONS}

The peak external knee adduction moment was decreased by the effect of 5-degree lateral wedge insole during walking condition for normal, overweight, and obese I participants. However, the peak external knee adduction moment did not have any effect upon the obese II group. The long-term effect and length of time for using lateral wedge insoles requires further research. The appropriate degree of lateral wedge insoles for reducing knee joint load among obese II individuals is of interest to further studies.

\section{ACKNOWLEDGEMENT}

1. This work was supported by the National Research Council of Thailand Graduated Student Fund [2014/6] and to be a part of the $\mathrm{PhD}$ thesis of the first author.

2. The Scholarship from the Graduate School, Chulalongkorn University to commemorate the $72^{\text {nd }}$ anniversary of his Majesty the King Bhumibala Aduladeja is gratefully acknowledged.

\section{REFERENCES}

[1] Jiang L, Rong J, Wang Y, Hu F, Bao C, Li X, et al. The relationship between body mass index and hip osteoarthritis: A systematic review and meta-analysis. Joint Bone Spine. 2011; 78(2): 150-5.

[2] Haslam DW, James WPT. Obesity. Lancet. 366(9492): 1197-209.

[3] Sangthong R, Wichaidit W, McNeil E, Chongsuvivatwong V, Chariyalertsak S, Kessomboon P, et al. Health behaviors among short- and long- term ex-smokers: Results from the Thai National Health Examination Survey IV, 2009. Prev Med. 2012; 55(1): 56-60.

[4] Zheng H, Chen C. Body mass index and risk of knee osteoarthritis: systematic review and meta-analysis of prospective studies. BMJ open. 2015; 5(12): e007568.

[5] Silverwood V, Blagojevic-Bucknall M, Jinks C, Jordan JL, Protheroe J, Jordan KP. Current evidence on risk factors for knee osteoarthritis in older adults: a systematic review and meta-analysis. Osteoarthritis Cartilage. 2015; 23(4): 507-15. 
[6] Nutalaya C, CHAIKUMARN M. Knee biomechanics in obese female during locomotion: a cross-sectional study. Journal of Sports Science Technology. 2017; 17(1): 21-35.

[7] Foroughi N, Smith RM, Lange AK, Baker MK, Fiatarone Singh MA, Vanwanseele B. Lower limb muscle strengthening does not change frontal plane moments in women with knee osteoarthritis: A randomized controlled trial. Clin Biomech. 2011; 26(2): 167-74.

[8] Koo S, Rylander JH, Andriacchi TP. Knee joint kinematics during walking influences the spatial cartilage thickness distribution in the knee. J Biomech. 2011; 44(7): 1405-9.

[9] Sheehan KJ, Gormley J. The influence of excess body mass on adult gait. Clin Biomech. 2013; 28(3): $337-43$.

[10] Henriksen M, Aaboe J, Bliddal H. The relationship between pain and dynamic knee joint loading in knee osteoarthritis varies with radiographic disease severity. A cross sectional study. Knee. 2012; 19(4): 392-8.

[11] Moyer RF, Birmingham TB, Dombroski CE, Walsh RF, Leitch KM, Jenkyn TR, et al. Combined effects of a valgus knee brace and lateral wedge foot orthotic on the external knee adduction moment in patients with varus gonarthrosis. Arch Phys Med Rehabil. 2013; 94(1): 103-12.

[12] Jenkyn TR, Erhart JC, Andriacchi TP. An analysis of the mechanisms for reducing the knee adduction moment during walking using a variable stiffness shoe in subjects with knee osteoarthritis. J Biomech. 2011; 44(7): 12716.

[13] Erhart JC, Mündermann A, Elspas B, Giori NJ, Andriacchi TP. A variable-stiffness shoe lowers the knee adduction moment in subjects with symptoms of medial compartment knee osteoarthritis. J Biomech. 2008; 41(12): 2720-5.

[14] Erhart JC, Mündermann A, Mündermann L, Andriacchi TP. Predicting changes in knee adduction moment due to load-altering interventions from pressure distribution at the foot in healthy subjects. J Biomech. 2008; 41(14): 2989-94.

[15] Fantini Pagani CH, Böhle C, Potthast W, Brüggemann G-P. Short-Term Effects of a Dedicated Knee Orthosis on Knee Adduction Moment, Pain, and Function in Patients With Osteoarthritis. Arch Phys Med Rehabil. 2010; 91(12): 1936-41.

[16] Radzimski AO, Mündermann A, Sole G. Effect of footwear on the external knee adduction moment - A systematic review. Knee. 2012; 19(3): 163-75.

[17] Russell EM, Hamill J. Lateral wedges decrease biomechanical risk factors for knee osteoarthritis in obese women. J Biomech. 2011; 44(12): 2286-91.

[18] Wilken JM, Rodriguez KM, Brawner M, Darter BJ. Reliability and minimal detectible change values for gait kinematics and kinetics in healthy adults. Gait Posture. 2012; 35(2): 301-7.

[19] Jones RK, Zhang M, Laxton P, Findlow AH, Liu A. The biomechanical effects of a new design of lateral wedge insole on the knee and ankle during walking. Hum Mov Sci. 2013; 32(4): 596-604.

[20] Cofré LE, Lythgo N, Morgan D, Galea MP. Aging modifies joint power and work when gait speeds are matched. Gait Posture. 2011; 33(3): 484-9.

[21] Tipnis RA, Anloague PA, Laubach LL, Barrios JA. The dose-response relationship between lateral foot wedging and the reduction of knee adduction moment. Clin Biomech. 2014; 29(9): 984-9.

[22] Barrios JA, Butler RJ, Crenshaw JR, Royer TD, Davis IS. Mechanical effectiveness of lateral foot wedging in medial knee osteoarthritis after 1 year of wear. J Orthop Res. 2013; 31(5): 659-64.

[23] Hinman RS, Bowles KA, Metcalf BB, Wrigley TV, Bennell KL. Lateral wedge insoles for medial knee osteoarthritis: Effects on lower limb frontal plane biomechanics. Clin Biomech. 2012; 27(1): 27-33.

[24] Kerrigan DC, Lelas JL, Goggins J, Merriman GJ, Kaplan RJ, Felson DT. Effectiveness of a lateral-wedge insole on knee varus torque in patients with knee osteoarthritis. Arch Phys Med Rehabil. 2002; 83(7): 889-93.

[25] Reilly KA, Barker KL, Shamley D. A systematic review of lateral wedge orthotics - how useful are they in the management of medial compartment osteoarthritis? Knee. 2006; 13(3): 177-83.

[26] Weinhandl JT, Sudheimer SE, Van Lunen BL, Stewart K, Hoch MC. Immediate and 1 week effects of laterally wedge insoles on gait biomechanics in healthy females. Gait Posture. 2016; 45: 164-9.

[27] Brisson NM, Stratford PW, Maly MR. Relative and absolute test-retest reliabilities of biomechanical risk factors for knee osteoarthritis progression: benchmarks for meaningful change. Osteoarthritis Cartilage. 2018; 26(2): 2206.

[28] Brisson NM, Stratford PW, Totterman S, Tamez-Peña JG, Beattie KA, Adachi JD, et al. Do knee moments normalized to measures of knee cartilage area better classify the severity of knee osteoarthritis? J Appl Biomech. 2015; 31(6): 415-22.

[29] Robbins SM, Astephen Wilson JL, Rutherford DJ, Hubley-Kozey CL. Reliability of principal components and discrete parameters of knee angle and moment gait waveforms in individuals with moderate knee osteoarthritis. Gait Posture. 2013; 38(3): 421-7.

[30] Robbins SMK, Birmingham TB, Maly MR, Chesworth BM, Giffin JR. Comparative diagnostic accuracy of knee adduction moments in knee osteoarthritis: A case for not normalizing to body size. J Biomech. 2011; 44(5): 96871. 